\title{
Coronary artery calcification is increased in patients with COPD and associated with increased morbidity and mortality
}

\author{
Michelle C Williams, ${ }^{1}$ John T Murchison, ${ }^{2}$ Lisa D Edwards, ${ }^{3}$ Alvar Agustí, ${ }^{4}$ Per Bakke, ${ }^{5}$ \\ Peter M A Calverley, ${ }^{6}$ Bartolome Celli, ${ }^{7}$ Harvey 0 Coxson, ${ }^{8}$ Courtney Crim, ${ }^{3}$ \\ David A Lomas, ${ }^{9}$ Bruce E Miller, ${ }^{10}$ Steve Rennard, ${ }^{11}$ Edwin K Silverman, ${ }^{7}$ \\ Ruth Tal-Singer, ${ }^{10}$ Jørgen Vestbo, ${ }^{12}$ Emiel Wouters, ${ }^{13}$ Julie C Yates, ${ }^{10}$ \\ Edwin J R van Beek, ${ }^{14}$ David E Newby, ${ }^{1}$ William MacNee, ${ }^{15}$ for the Evaluation of \\ COPD Longitudinally to Identify Predictive Surrogate Endpoints (ECLIPSE) investigators
}

- Additional material is published online only. To view please visit the journal online (http://dx.doi.org/10.1136/ thoraxjnl-2012-203151).

For numbered affiliations see end of article.

\section{Correspondence to} Dr Michelle C Williams, University/BHF Centre for Cardiovascular Science, Chancellor's Building, SU305, 49 Little France Crescent, Edinburgh EH16SUF, UK; michelle.williams@ed.ac.uk

Received 19 December 2012 Revised 18 November 2013 Accepted 21 December 2013 Published Online First 28 January 2014

CrossMark

To cite: Williams MC Murchison JT, Edwards LD, et al. Thorax 2014;69: 718-723.

\section{ABSTRACT}

Background Coronary artery calcification is pathognomonic of coronary artery disease (CAD). Whether CAD in patients with COPD is linked to lung function, functional capacity and/or clinically relevant outcomes is unknown. The objective was to assess the association between CAD and disease severity, functional capacity and outcomes in patients with COPD.

Methods Coronary artery calcium score (CACS; Agatston score) was measured using chest $C T$ in patients with COPD, smokers with normal spirometry and nonsmokers from the Evaluation of COPD Longitudinally to Identify Predictive Surrogate Endpoints (ECLIPSE) study.

Results CACS was measured in 942 subjects: 672 with COPD (mean age $\pm S D, 63 \pm 7$ years; FEV $149 \pm 16 \%$ predicted), 199 smokers with normal spirometry (54 \pm 9 years; $F E V_{1} 110 \pm 12 \%$ predicted) and 71 nonsmokers ( $55 \pm 9$ years; FEV $114 \pm 14 \%$ predicted). CACS was higher in patients with COPD than smokers or non-smokers (median (IQR), 128 (492) vs 0 (75) vs 0 (3) Agatston units $(A U), p<0.001)$. In patients with COPD, CACS correlated with age, pack-years, 6 min walking distance, modified Medical Research Council Dyspnoea score and circulating levels of interleukin (IL)-6, IL-8, Clara Cell protein 16, surfactant protein $D$ and peripheral blood neutrophil count, but not with emphysema, exacerbation frequency, \% predicted $\mathrm{FEV}_{1}$ or decline in $\mathrm{FEV}_{1}$. CACS was higher in patients with COPD who died than in those who survived until 3-year follow-up (CACS 406 vs $103 \mathrm{AU}, p<0.001$ ), and was associated with mortality in a Cox proportional hazards model ( $p=0.036)$.

Conclusions Patients with COPD have more CAD than controls and this is associated with increased dyspnoea, reduced exercise capacity and increased mortality. These data indicate that the presence of CAD in patients with COPD is associated with poor clinical outcomes.

\section{INTRODUCTION}

The relationship between COPD and coronary artery disease (CAD) is complex and remains poorly understood. Both diseases have a high worldwide prevalence and frequently coexist. ${ }^{1}$ COPD is an independent risk factor for CAD and

\section{Key messages}

What is the key question?

- What is the relationship between coronary artery calcification and clinical outcomes in patients with COPD?

\section{What is the bottom line?}

- The extent of coronary artery calcification was substantially greater in patients with COPD than in smokers with normal spirometry or non-smokers, and was correlated with dyspnoea, exercise capacity and all-cause mortality, but not with FEV or exacerbations of COPD, indicating a link between coronary artery calcification and poor clinical outcome in COPD.

\section{Why read on?}

- The identification of patients with a high coronary artery calcium score is important to provide appropriate targeted therapy to modify cardiovascular risk by treating cardiovascular disease appropriately.

the presence of COPD is associated with an increased risk of CAD and cardiovascular mortality. ${ }^{1}$ In the Evaluation of COPD Longitudinally to Identify Predictive Surrogate Endpoints (ECLIPSE) study (SCO104960; NCT00292552), patients with COPD had an increased prevalence of self-reported CAD compared with smoker and non-smoker control subjects. ${ }^{3}$ However, this increased prevalence is not solely attributable to shared risk factors, with COPD remaining an independent predictor for the development of CAD after adjustment for smoking and other cardiovascular risk factors. ${ }^{4}$

Coronary artery calcification can be identified by CT and is pathognomonic of coronary atherosclerosis. Coronary artery 'calcium scoring' methods, such as the Agatston score, quantify this calcification based on its extent and density on CT images. 
Coronary artery calcium score (CACS) is widely used to identify and stratify risk for CAD. ${ }^{5}$ CACS correlates with increased mortality in smokers ${ }^{6}$ and may provide additive prognostic information to that obtained from traditional methods of cardiovascular risk stratification such as the Framingham risk score. ${ }^{7}$

The pathogenic mechanisms linking COPD and CAD remain unknown, but are likely to involve a multifactorial process of systemic inflammation, oxidative stress, increased thrombogenicity, vascular dysfunction and hypoxia. ${ }^{4}{ }^{8-10}$ Although lowgrade systemic inflammation occurs in a variety of conditions, including COPD and CAD,${ }^{11-13}$ the weak associations identified between inflammatory markers and $\mathrm{CACS}^{14}$ or atherosclerotic plaque burden ${ }^{15}$ suggest that the mechanisms of association may be distinct.

In patients with COPD, the relationship between CACS and clinically relevant long-term outcomes such as mortality, exacerbation rate or $\mathrm{FEV}_{1}$ decline have not previously been evaluated. The aims of this study were to assess these associations in the ECLIPSE study cohort.

\section{METHODS}

\section{Study population and ethics}

We assessed coronary artery calcium on non-gated chest CT images in a subset of subjects recruited to the ECLIPSE study. ${ }^{16}$ ECLIPSE is an international multicentre observational longitudinal cohort study of subjects with COPD, smokers with normal spirometry and non-smokers. Ethics committees of all participating institutions approved the study and written informed consent was obtained from all subjects.

Participants were aged 40-75 years and presence of significant lung disease was determined by history, physical examination and screening investigations. Patients with COPD were current or ex-smokers $(\geq 10$ pack years), with baseline postbronchodilator $\mathrm{FEV}_{1}<80 \%$ of the reference value and $\mathrm{FEV}_{1}$ / FVC $<0.7$. Smokers with normal spirometry were free of significant lung disease, were current or ex-smokers ( $\geq 10$ pack years), and had baseline post-bronchodilator $\mathrm{FEV}_{1}>85 \%$ and $\mathrm{FEV}_{1}$ / FVC $>0.7$. Non-smokers were free from significant lung disease, had smoking history of $<1$ pack year, and had baseline postbronchodilator $\mathrm{FEV}_{1}>85 \%$ and $\mathrm{FEV}_{1} / \mathrm{FVC}>0.7$. Key exclusion criteria were the presence of respiratory disorders other than COPD and a reported COPD exacerbation within 4 weeks of enrolment. Comorbidities were not excluded, but these had to be clinically stable at the time of assessment.

\section{Clinical, physiological and biochemical measurements}

Participants underwent detailed phenotypic characterisation and follow-up over 3 years, including lung physiology, imaging, biomarkers, health outcomes and mortality, as reported previously ${ }^{3}$ (see online supplement).

\section{Computed tomography}

All subjects underwent a low-dose CT of the chest using multidetector scanners with a minimum of four rows (GE Healthcare or Siemens Healthcare). Imaging was performed in the supine position, at suspended full inspiration, without administration of intravenous contrast. Exposure settings were $120 \mathrm{kVp}$ and $40 \mathrm{mAs}$ and images were reconstructed using $1.0 \mathrm{~mm}$ (Siemens) or $1.25 \mathrm{~mm}$ (GE) contiguous slices and a low spatial frequency reconstruction algorithm (GE: Standard; Siemens: b35f). Quantitative assessment of the percentage of low attenuation areas (\%LAA), representing the presence of emphysema, was performed using a threshold of -950 Hounsfield units.

\section{Coronary artery calcium score}

CACS was assessed on the standard images used for analysis of the lungs and no additional reconstructions were performed. CT scans were assessed by a trained observer (MCW), who was blinded to subject characteristics. One hundred randomly selected scans were reassessed (by MCW and JM) to assess intra-observer and inter-observer variability. Images were analysed on a dedicated workstation using dedicated analysis software (VScore, Vitrea Fx, V.3.1.0, Vital Images, Minnetonka, Minnesota, USA).

Image quality was assessed on a five-point Likert scale (1, excellent; 2 , mild artefact; 3 , moderate artefact; 4 , severe artefact; 5, non-diagnostic) for each of the three main coronary arteries. Image quality scores from individual vessels were summed to calculate the total image quality score. Images with a total image quality score of 15 were excluded from further analysis.

CACS was assessed using the Agatston scoring method. ${ }^{17}$ Coronary calcification was defined as an area in the course of a coronary artery that had an attenuation threshold of $\geq 130$ Hounsfield units and was $\geq 1 \mathrm{~mm}^{2}$. On each axial slice the area of calcification that met these requirements was measured and then multiplied by a weighting factor dependent on the peak attenuation within the region. These weighted areas were summed to produce the total Agatston CACS. For the interpretation of Agatston score in asymptomatic patients the absolute value can be used or it can be considered as low $(<100$ Agatston units (AU)), intermediate (101-400 AU), high (401$1000 \mathrm{AU})$ or very high (>1000 AU).

Normal ranges for Agatston scores in healthy asymptomatic individuals stratified by age, gender and ethnicity have been published. ${ }^{18}$ These can be used to calculate the CACS percentile (CACSP) for individual patients. A CACSP above 90\% can be used as an indication to commence primary preventative therapy.

\section{Statistical analysis}

Results are shown as mean (SD), median (IQR), frequency distribution or proportions. Comparisons between subject groups were carried out using analysis of variance, Kruskal-Wallis tests, van Elteren tests or Cochran-Mantel-Haenszel tests, adjusting for age, gender and smoking pack-years. Pack-year-adjusted CACSP medians and IQRs were calculated from quantile regression models based on 1000 bootstrap samples. Linear associations were assessed by Spearman correlation coefficients due to non-normality of the CACS and CACSP; 95\% CIs were calculated by the Fisher z-transformation method. To adjust for effects of covariates on CACS, multiple regression was conducted on the log-transformed values; estimates were backtransformed for presentation. The CACSP did not respond to $\log$ transformation, so the analysis was two-stage; a logistic regression model to describe the effect of covariates on a zero versus non-zero percentile and multiple regression on the logtransformed non-zero values. Cox proportional hazard models were constructed to compare mortality between subject groups. For all models, covariates that were expected to impact the dependent variables or showed differences among the cohorts were included, including age, sex and pack-years. Two-sided $\mathrm{p}<0.05$ values were considered significant with no adjustment for multiple testing. Analyses were conducted with SAS V.9.1 (SAS Institute, Cary, North Carolina, USA). Intra-observer and inter-observer agreement were assessed via concordance correlation coefficients. ${ }^{19}$ 
Table 1 Demographic and baseline details of the study population

\begin{tabular}{|c|c|c|c|}
\hline \multirow[b]{2}{*}{ Characteristic } & \multicolumn{3}{|c|}{ Study cohort } \\
\hline & $\begin{array}{l}\text { COPD } \\
\text { subjects }\end{array}$ & Smokers & Non-smokers \\
\hline $\mathrm{N}$ & 672 & 199 & 71 \\
\hline Age years & $63.2(7.0)$ & $54.1(8.5)^{*}$ & $54.7(9.2)^{*}$ \\
\hline Men & $422(63 \%)$ & $115(58 \%)$ & $21(30 \%)^{*}$ \\
\hline Pack-years & $47.4(25.1)$ & $30.7(19.9)^{*}$ & $0.1(1.0)^{*}$ \\
\hline Body mass index, $\mathrm{kg} / \mathrm{m}^{2}$ & $26.5(5.6)$ & $26.7(4.3)$ & $28.3(6.9)^{*}$ \\
\hline $\begin{array}{l}\text { Post-bronchodilator FEV }{ }_{1} \text {, } \\
\text { litres }\end{array}$ & $1.36(0.54)$ & $3.46(0.71)^{*}$ & $3.19(0.79)^{*}$ \\
\hline $\begin{array}{l}\text { Post-bronchodilator } \mathrm{FEV}_{1} \% \\
\text { predicted }\end{array}$ & $48.7(16.1)$ & $110.0(11.5)^{*}$ & $114.4(13.8)^{*}$ \\
\hline $\begin{array}{l}\text { Post-bronchodilator } \mathrm{FEV}_{1} / \mathrm{FVC} \\
\text { ratio }\end{array}$ & $44.1(11.5)$ & $79.2(4.8)^{*}$ & $81.0(5.9)^{*}$ \\
\hline $\begin{array}{l}\text { Patient-reported } \\
\text { cardiovascular disease }\end{array}$ & $357(53 \%)$ & $45(23 \%)^{*}$ & $24(34 \%)^{*}$ \\
\hline$\%$ LAA & $15.6[19.2]$ & $0.8[1.9]^{*}$ & $2.8[4.4]^{*}$ \\
\hline
\end{tabular}

\section{RESULTS}

\section{Demographic details}

Of the 2746 subjects enrolled in ECLIPSE, 2574 patients had scans available for analysis. From a random sample of 1001 scans, CACS was measured in 942 scans after the exclusion of duplicate scans and five scans $(0.5 \%)$ with poor image quality (image quality score of 15). The demographic details of the study population are show in table 1 and differences between the ECLIPSE cohort and CACS cohort are shown in online supplement table Si. Consistent with the total ECLIPSE cohort, subjects with COPD were older, had a higher pack-year history, a lower post-bronchodilator \% predicted $\mathrm{FEV}_{1}$ and a higher \% LAA. Patients with COPD also had a higher frequency of diabetes mellitus, hypertension, statin therapy and patient-reported cardiovascular disease than smoker or non-smoker controls (table 2).

\section{Coronary artery calcium score}

Intra-observer and inter-observer agreement for CACS were excellent (concordance correlation coefficients 0.992 and 0.956, respectively, $\mathrm{p}<0.001$ for both).
The median CACS was higher in subjects with COPD than control smokers and non-smokers (128 (492) vs 0 (75) vs 0 (3) AU, $p<0.001$, online supplement table Sii). The CACS was also higher in men than women and in those who reported cardiovascular disease, diabetes mellitus, hypertension or treatment with statin therapy (see online supplement tables Siii-Sv). The absence of coronary artery calcification was more frequent in non-smokers $(72 \%)$ than for smokers $(53 \%)$ or patients with COPD (23\%). High (>400 AU) or very high (>1000 AU) CACS were more frequent in patients with COPD (see online supplement table Svi).

The CACSP was greater in patients with COPD than in smokers or non-smokers $(71 \%(60 \%)$ vs $0(78 \%)$ vs $0(43 \%)$, respectively, $\mathrm{p}<0.001)$. When further corrected for pack-years, the CACSP remained greater in patients with COPD than in smokers and non-smokers.

Spearman correlation analysis showed that CACS in patients with COPD was associated with age and pack-years (see online supplement table Svii). CACS also correlated with markers of functional capacity and symptoms, including the 6 min walking distance, modified Medical Research Council Dyspnoea score and BODE index. ${ }^{20}$ CACS in patients with COPD was weakly correlated with the extent of emphysema and with borderline statistical significance and with $\mathrm{FEV}_{1} / \mathrm{FVC}$ ratio. However, there were no significant correlations with $\mathrm{FEV}_{1}, \mathrm{FEV}_{1} \%$ predicted, decline in $\mathrm{FEV}_{1}$ or reported exacerbation frequency. When stratified into the four patient types proposed by the 2011 Global Initiative for Chronic Obstructive Lung Disease (GOLD) strategy document (A, B, C and D), ${ }^{21}$ there were no differences in CACS $(p=0.681)$ or CACSP $(p=0.398)$.

CACS in subjects with COPD correlated with several circulating inflammatory biomarkers (see online supplement table Svii), including interleukin (IL)-6, IL-8, Clara cell secretory protein 16 , surfactant protein D and peripheral blood neutrophil count. It did not correlate with high-sensitivity C-reactive protein (CRP), fibrinogen, tumour necrosis factor $\alpha$, chemokine ligand 18, sRAGE or total white blood cell count. There was a higher CACS and CACSP in patients with persistent systemic inflammation (defined as two or more elevated biomarkers on two occasions) ${ }^{12}$ compared with patients who had no elevated biomarkers (see online supplement table Sviii). This difference was statistically significant for CACS but not CACSP.

\section{Multivariate analysis of CACS and percentiles}

Multiple regression analysis showed that CACS score was associated with age, gender, pack-years, \% predicted $\mathrm{FEV}_{1}$ and patient-reported cardiovascular disease. CACSP was associated

Table 2 Cardiovascular risk factors

\begin{tabular}{|c|c|c|c|c|c|c|c|}
\hline \multirow[b]{2}{*}{ Location } & \multirow[b]{2}{*}{$\begin{array}{l}\text { Patients with } \\
\text { COPD }\end{array}$} & \multirow[b]{2}{*}{ Smokers } & \multirow[b]{2}{*}{ Non-smokers } & \multicolumn{4}{|l|}{$\mathrm{p}$ Value } \\
\hline & & & & Overall & $\begin{array}{l}\text { Patients with } \\
\text { COPD vs smokers }\end{array}$ & $\begin{array}{l}\text { Patients with } \\
\text { COPD vs non-smokers }\end{array}$ & $\begin{array}{l}\text { Smokers vs } \\
\text { non-smokers }\end{array}$ \\
\hline Diabetes & $66(10 \%)$ & $14(7 \%)$ & $3(4 \%)$ & 0.158 & 0.213 & 0.115 & 0.402 \\
\hline Hypertension & $260(41 \%)$ & $31(17 \%)$ & $17(25 \%)$ & $<0.001$ & $<0.001$ & 0.015 & 0.115 \\
\hline Statin therapy & $173(26 \%)$ & 27 (14\%) & $8(11 \%)$ & $<0.001$ & $<0.001$ & 0.007 & 0.621 \\
\hline $\begin{array}{l}\text { Patient-reported cardiovascular } \\
\text { disease }^{*}\end{array}$ & $357(53 \%)$ & $45(23 \%)$ & $24(34 \%)$ & $<0.001$ & $<0.001$ & 0.002 & 0.064 \\
\hline
\end{tabular}


Table 3 Multivariate analysis for calcium score and coronary artery calcium score percentile (CACSP) based on age, gender and ethnicity

\begin{tabular}{|c|c|c|c|c|c|c|}
\hline \multirow[b]{2}{*}{ Parameter } & \multicolumn{3}{|l|}{ Agatston score } & \multicolumn{3}{|c|}{ CACSP based on age, gender and ethnicity } \\
\hline & Effect estimate* (SE) & p Value & Model $\mathbf{R}^{2}$ & Effect Estimate* (SE) & p Value & Model $\mathbf{R}^{2}$ \\
\hline Age (per 10 years) & $4.67(1.18)$ & $<0.001$ & 0.242 & $0.88(1.02)$ & $<0.001$ & 0.153 \\
\hline Women & $0.51(1.27)$ & 0.005 & & $1.20(1.03)$ & $<0.001$ & \\
\hline Pack-years (per 20 years) & $1.22(1.09)$ & 0.026 & & $0.99(1.01)$ & 0.320 & \\
\hline $\mathrm{FEV}_{1} \%$ predicted (post bronchodilator) (per 10\%) & $0.85(1.08)$ & 0.029 & & $1.01(1.01)$ & 0.590 & \\
\hline CVD & $2.37(1.25)$ & $<0.001$ & & $1.06(1.03)$ & 0.046 & \\
\hline 6MWD (per $100 \mathrm{~m}$ ) & $1.00(1.10)$ & 0.990 & & $0.97(1.01)$ & 0.017 & \\
\hline IL-6 (per 1 SD) & $0.99(1.11)$ & 0.953 & & $1.02(1.02)$ & 0.221 & \\
\hline Clara cell protein (per 1 SD) & $1.01(1.12)$ & 0.951 & & $1.02(1.02)$ & 0.132 & \\
\hline Surfactant protein D (per 1 SD) & $1.15(1.12)$ & 0.199 & & $1.02(1.02)$ & 0.177 & \\
\hline
\end{tabular}

with age, gender, patient-reported cardiovascular disease and the 6 min walking distance (table 3).

\section{CACS and mortality}

Patients with COPD who died $(n=63)$ during 3 years of follow-up had a higher CACS, CACSP and CACSP adjusted for pack-years than subjects who survived (see online supplement table Six). As a single predictor of mortality, CACS had a HR of 1.55 (95\% CI 1.26 to 1.92 ) and CACSP greater than $90 \%$ had a HR of 1.66 (95\% CI 0.98 to 2.81). After adjustment for age, gender and pack-years, CACS had a HR of 1.42 (CI 1.12 to 1.78) and CACSP had a HR of 1.77 (95\% CI 1.04 to 3.01). In Cox proportional hazards models the CACS adjusted for age,
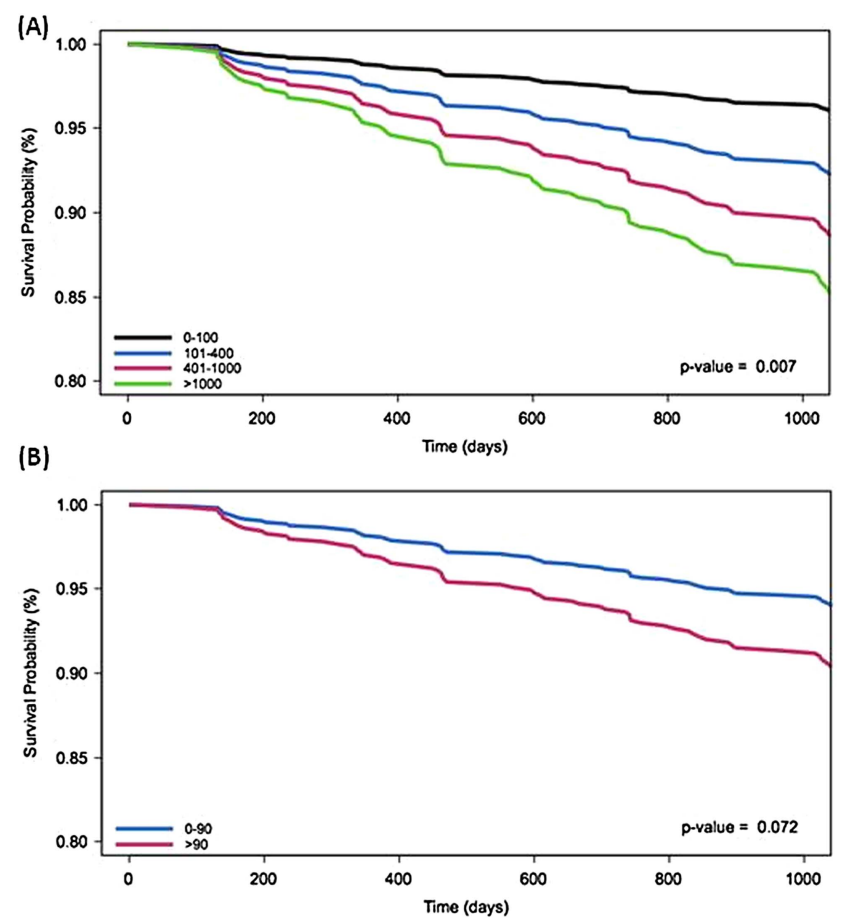

Figure 1 Cox proportional hazards model for patients with COPD and (A) coronary artery calcium score (CACS) (Agatston score)adjusted for age, gender, pack-years, severity of COPD and self-reported cardiovascular disease and (B) CACSP adjusted for pack-years. gender and pack-years was associated with increased mortality in all subjects $(\mathrm{p}=0.005)$ and in patients with COPD $(\mathrm{p}=0.007)$. The same was found for a CACSP greater than $90 \%$ $(\mathrm{p}=0.051$ for all subjects, and $\mathrm{p}=0.072$ for patients with COPD) (figure 1 and online supplement figure $\mathrm{Si}$ ). The addition of hypertension or statin therapy had little effect on the HRs and neither was significant in the models. CACS and patientreported cardiovascular disease were predictive of mortality, but the two assessments together did not improve the prediction of mortality.

\section{DISCUSSION}

In a large cohort of well characterised subjects, we have shown that patients with COPD had a higher CACS corrected for age, gender, ethnicity and pack-year smoking history than smokers with normal spirometry or non-smokers. Moreover, a higher CACS is associated with adverse functional capacity and increased mortality in these patients. We conclude that patients with COPD have a disproportionately greater burden of CAD that causes increased morbidity and mortality.

Low-level chronic inflammation is believed to play an important role in COPD and cardiovascular disease. ${ }^{12}$ Coronary artery calcium and atherosclerotic plaque burden are associated with increased systemic inflammatory markers such as CRP, IL-6 and fibrinogen in population studies. ${ }^{14}{ }^{15}$ However, in our study these associations were weak, suggesting that inflammatory markers and coronary artery calcium may reflect different disease mechanisms. Recently a pattern of systemic inflammation with raised levels of CRP, IL-6, white blood cell count and fibrinogen has been associated with COPD. ${ }^{12}$ Persistent inflammation (defined as elevation in two systemic biomarkers) occurred in a proportion of patients who had significantly worse outcomes. ${ }^{12}$ In our study the systemic inflammatory phenotype was associated with a small increase in CACS. We therefore believe that it is unlikely that inflammation alone can account for the association between COPD and CAD.

In the multiple regression analysis CACS was associated with the degree of airflow limitation. However, whether lung disease per se has a direct influence on the initiation or progression of CAD remains unclear. Due to overlap in the diagnostic symptoms, it can be difficult to establish whether CAD is present in patients with COPD. Indeed, acute coronary syndromes and stable angina are under-diagnosed in patients with COPD. ${ }^{22-24}$ 
The association between CACS and dyspnoea, quality of life and exercise capacity suggests that some symptoms and functional impairment may be attributable to occult CAD rather than COPD. Thus, the identification of coronary artery calcification by CT may aid in the diagnosis and treatment of occult CAD in patients with COPD.

Cardiovascular disease is an important cause of morbidity and mortality in patients with COPD, accounting for up to $50 \%$ of deaths. $^{25}$ Furthermore, patients with more severe COPD have higher cardiovascular morbidity and mortality. ${ }^{1}$ This association may indicate a shared pathogenesis, a synergistic impact on disease progression or an under diagnosis or under treatment of concomitant CAD in patients with COPD. In addition, there may be common responses to smoking-induced tissue injury, such as inflammation, oxidative stress or tissue remodelling. ${ }^{26}$ The association between CACS and cardiovascular events or mortality has been established in asymptomatic individuals, ${ }^{27-29}$ subjects at intermediate ${ }^{30}$ or high $^{6}$ likelihood of cardiovascular disease, and patients with hypertension ${ }^{31}$ or diabetes mellitus. ${ }^{32}$ An association between CACS and mortality has also been identified in lung cancer screening studies. ${ }^{33}$ Our results extend these findings to show, for the first time, that CACS is also associated with increased morbidity and mortality in patients with COPD. Clinical intervention trials are now warranted to ascertain whether screening and treatment for occult CAD may improve the clinical outcome of patients with COPD.

\section{Study limitations}

Coronary artery calcification is a surrogate marker of coronary atherosclerosis and a higher CACS is associated with increased risk of significant coronary artery stenosis. ${ }^{34}$ However, in the absence of coronary artery calcification, haemodynamically significant non-calcified plaques may be present in between $0.8 \%$ and $0.9 \%$ of asymptomatic individuals. ${ }^{35}$ Thus a zero calcium score in our study is likely to be associated with an underestimation of the prevalence of CAD.

The standard reconstruction parameters for calcium scoring utilises $3 \mathrm{~mm}$ non-overlapping slices. ${ }^{36}$ However, coronary artery calcium can be identified using non-gated chest CT images. $^{33}{ }^{37}$ Good correlations have been identified between standard reconstructions and CACS calculated using chest $\mathrm{CT}^{38}$ or non-gated images. ${ }^{37}$ However, the absolute Agatston numbers may not be the same as those calculated using standard reconstructions. Nevertheless, this technique can be used to investigate associations between subgroups within this study.

Cigarette smoking is a major and common risk factor for COPD and CAD. Any association between COPD and CAD could therefore be explained by this shared risk factor. In our study patients with COPD had higher pack-years than smoking controls. We adjusted for linear effects of pack-years in the regression models, but there could be non-linear effects that could contribute to the observed differences.

A further limitation is that cardiovascular events were not recorded and cardiovascular risk scores such as the Framingham risk score could not be calculated. Confounding factors such as hypertension and hyperlipidaemia could also affect the study results, as could other unmeasured cardiovascular risk factors. The ECLIPSE study excluded patients with respiratory disorders other than COPD and thus these results are not necessarily generalisable to other respiratory disorders. Participants with acute exacerbations within 4 weeks of enrolment were also excluded. However, coronary artery calcium develops over a prolonged period of time, thus this is unlikely to affect the results.

\section{CONCLUSION}

This is the first large cohort study to identify the presence of a higher CACS in patients with COPD and to show an association of this with poor clinical outcomes in these patients, including all-cause mortality.

\section{Author affiliations}

${ }^{1}$ University of Edinburgh/British Heart Foundation Centre for Cardiovascular Science, Edinburgh, UK

${ }^{2}$ Royal Infirmary of Edinburgh, Edinburgh, UK

${ }^{3}$ GlaxoSmithKline, Research Triangle Park, North Carolina, USA

${ }^{4}$ Thorax Institute, Hospital Clinic, Universitat de Barcelona; CIBER Enfermedades Respiratorias (CIBERES), FISIB, Mallorca, Spain

${ }^{5}$ University of Bergen, Bergen, Norway

${ }^{6}$ Department of Respiratory Medicine, University Hospital Aintree, Liverpool, UK

${ }^{7}$ Department of Respiratory Medicine, Brigham and Women's Hospital, and Harvard

Medical School, Boston, Massachusetts, USA

${ }^{8}$ University of British Columbia, Vancouver, British Columbia, Canada

${ }^{9}$ Department of Medicine, University of Cambridge, Cambridge, UK

${ }^{10} \mathrm{GlaxoSmithKline,} \mathrm{King} \mathrm{of} \mathrm{Prussia,} \mathrm{Pennsylvania,} \mathrm{USA}$

${ }^{11}$ University of Nebraska Medical Center, Omaha, Nebraska, USA

${ }^{12}$ Department of Respiratory Medicine, Odense University \& University of Southern

Denmark, Denmark/University of Manchester, Academic Health Science Centre,

Manchester, UK

${ }^{13}$ Department of Respiratory Medicine, Maastricht University Medical Centre,

Maastricht, The Netherlands

${ }^{14}$ Clinical Research Imaging Centre, University of Edinburgh, Edinburgh, UK

${ }^{15}$ Centre for Inflammation Research, University of Edinburgh, Edinburgh, UK

Acknowledgements We thank the $\mathrm{CT}$ analysis staff (T Candido, S Cogswell, $\mathrm{H}$ Davis, N Farzaneh, L Holy, N Krowchuk, H Lee, E Phillips, C Storness-Bliss, N Tai, A-T Tran, N Tran, E Wang, and T Yokogawa) for technical assistance with the CT analysis and data management.

Collaborators See online supplementary appendix for the principal investigators and centres participating in ECLIPSE.

Contributors The study was conceived and conducted by WM, AA, PB, PMAC, BC, HOC, CC, DAL, BEM, SR, EKS, RT-S, JV, EW and JCY with additional patient recruitment from the Longitudinally to Identify Predictive Surrogate Endpoints (ECLIPSE) study investigators. Images were assessed by MCW, JTM and EvB. Data were analysed by MCW, LDE and WM. The manuscript was prepared by MCW, WM, JTM, DEN and LDE. Critical revision of the manuscript was provided by AA, PB, PMAC, BC, HOC, CC, DAL, BEM, SR, EKS, RT-S, EvB, JV, EW and JCY. MCW and WM were responsible for the overall content of the manuscript as guarantors.

Funding GlaxoSmithKline. MCW is supported by a British Heart Foundation Clinical Research and Training Fellowship (FS/11/14/28692) and DEN holds the British Heart Foundation John Wheatley Chair of Cardiology (CH/09/002). The Centre for Cardiovascular Science is the recipient of a British Heart Foundation Centre of Research Excellence Award (RE/08/001). The Clinical Research Imaging Centre is supported by NHS Research Scotland (NRS) through NHS Lothian.

Competing interests The ECLIPSE study was supported by GSK.

Patient consent Obtained.

Ethics approval Ethical approval from institutional ethics committees in each of the centres.

Provenance and peer review Not commissioned; externally peer reviewed.

\section{REFERENCES}

1 Curkendall SM, Lanes $S$, de Luise $C$, et al. Chronic obstructive pulmonary disease severity and cardiovascular outcomes. Eur J Epidemiol 2006;21:803-13.

2 Mannino DM, Thorn D, Swensen A, et al. Prevalence and outcomes of diabetes, hypertension and cardiovascular disease in COPD. Eur Respir J 2008;32:962-9.

3 Agusti A, Calverley PM, Celli B, et al. Characterisation of COPD heterogeneity in the ECLIPSE cohort. Respir Res 2010;11:122.

4 Boschetto $P$, Beghé $B$, Fabbri LM, et al. The link between COPD and coronary artery disease-implication for clinical practice. Respirology 2012;17:422-31.

5 Greenland $\mathrm{P}$, Bonow RO, Brundage BH, American College of Cardiology Foundation Clinical Expert Consensus Task Force (ACCF/AHA Writing Committee to Update the 2000 Expert Consensus Document on Electron Beam Computed Tomography), Society of Atherosclerosis Imaging and Prevention, Society of Cardiovascular Computed Tomography, et al. ACCF/AHA 2007 clinical expert consensus document on coronary artery calcium scoring by computed tomography in global cardiovascular risk assessment and in evaluation of patients with chest pain. $J$ Am Coll Cardiol 2007;115:402-26. 
6 Jacobs PC, Prokop M, van der Graaf $Y$, et al. Comparing coronary artery calcium and thoracic aorta calcium for prediction of all-cause mortality and cardiovascular events on low-dose non-gated computed tomography in a high-risk population of heavy smokers. Atherosclerosis 2010;209:455-62.

7 Okwuosa TM, Greenland P, Ning H, et al. Distribution of coronary artery calcium scores by Framingham 10-year risk strata in the MESA (Multi-Ethnic Study of Atherosclerosis) potential implications for coronary risk assessment. J Am Coll Cardiol 2011:57:1838-45.

8 Maclay JD, McAllister DA, Rabinovich $R$, et al. Systemic elastin degradation in chronic obstructive pulmonary disease. Thorax 2012;67:606-12.

9 MacNee W, Maclay J, McAllister D. Cardiovascular injury and repair in chronic obstructive pulmonary disease. Proc Am Thorac Soc 2008;5:824-33.

10 Mills NL, Miller JJ, Anand A, et al. Increased arterial stiffness in patients with chronic obstructive pulmonary disease: a mechanism for increased cardiovascular risk. Thorax 2008;63:306-11.

11 Fabbri LM, Rabe KF. From COPD to chronic systemic inflammatory syndrome? Lancet 2007;370:797-9.

12 Agustí A, Edwards LD, Rennard SI, Evaluation of COPD Longitudinally to Identify Predictive Surrogate Endpoints (ECLIPSE) Investigators, et al. Persistent systemic inflammation is associated with poor clinical outcomes in COPD: a novel phenotype. PloS ONE 2012;7:e37483.

13 Thomsen $\mathrm{M}$, Dahl $\mathrm{M}$, Lange $\mathrm{P}$, et al. Inflammatory biomarkers and comorbidities in chronic obstructive pulmonary disease. Am J Respir Crit Care Med 2012;186:982-8

14 Jenny NS, Brown ER, Detrano R, et al. Associations of inflammatory markers with coronary artery calcification: results from the Multi-Ethnic Study of Atherosclerosis. Atherosclerosis 2010;209:226-9.

15 Lin T, Liu J-C, Chang L-Y, et al. Association of C-reactive protein and homocysteine with subclinical coronary plaque subtype and stenosis using low-dose MDCT coronary angiography. Atherosclerosis 2010;212:501-6.

16 Vestbo J, Anderson W, Coxson HO, ECLIPSE investigators, et al. Evaluation of COPD Longitudinally to Identify Predictive Surrogate End-points (ECLIPSE). Eur Respir J 2008;31:869-73.

17 Agatston AS, Janowithz WR, Hildner FJ, et al. Quantification of coronary artery calcium using ultrafast computed tomography. J Am Coll Cardiol 1990;15:827-32.

18 McClelland RL, Chung H, Detrano R, et al. Distribution of coronary artery calcium by race, gender, and age: results from the Multi-Ethnic Study of Atherosclerosis (MESA). Circulation 2006:113:30-7.

19 Lin LI. A concordance correlation coefficient to evaluate reproducibility. Biometrics 1989;45:255-68

20 Celli BR, Cote CG, Marin JM, et al. The body-mass index, airflow obstruction, dyspnea, and exercise capacity index in chronic obstructive pulmonary disease. $N$ Engl J Med 2004;350:1005-12.

21 Vestbo J, Hurd SS, Agusti AG, et al. Global strategy for the diagnosis, management and prevention of chronic obstructive pulmonary disease, GOLD executive summary. Am J Respir Crit Care Med 2012;187:347-65.

22 Gunes $Y$, Tuncer $M$, Guntekin $U$, et al. Reliability of symptoms suggestive of angina in patients with chronic obstructive pulmonary disease. Arq Bras Cardiol 2009;92:334-8.
23 Brekke PH, Omland T, Smith $\mathrm{P}$, et al. Underdiagnosis of myocardial infarction in COPD—Cardiac Infarction Injury Score (CIIS) in patients hospitalised for COPD exacerbation. Respir Med 2008:102:1243-7.

24 McAllister DA, Maclay JD, Mills NL, et al. Diagnosis of myocardial infarction following hospitalisation for exacerbation of COPD. Eur Respir $J$ 2012;39:1097-103.

25 Camilli AE, Robbins DR, Lebowitz MD. Death certificate reporting of confirmed airways obstructive disease. Am J Epidemiol 1991;133:795-800.

26 Maclay JD, MacNee W. Cardiovascular disease in COPD: mechanisms. Chest 2013:143:798-807.

27 Pletcher MJ, Tice JA, Pignone M, et al. Using the coronary artery calcium score to predict coronary heart disease events: a systematic review and meta-analysis. Arch Intern Med 2004;164:1285-92.

28 Taylor AJ, Bindeman J, Feuerstein I, et al. Coronary calcium independently predicts incident premature coronary heart disease over measured cardiovascular risk factors: mean three-year outcomes in the Prospective Army Coronary Calcium (PACC) project. J Am Coll Cardiol 2005;46:807-14.

29 Vliegenthart R, Oudkerk M, Hofman A, et al. Coronary calcification improves cardiovascular risk prediction in the elderly. Circulation 2005;112:572-7.

30 Schenker MP, Dorbala S, Hong ECT, et al. Interrelation of coronary calcification, myocardial ischemia, and outcomes in patients with intermediate likelihood of coronary artery disease: a combined positron emission tomography/computed tomography study. Circulation 2008;117:1693-700.

31 Shemesh J, Morag-Koren N, Goldbourt U, et al. Coronary calcium by spiral computed tomography predicts cardiovascular events in high-risk hypertensive patients. J Hypertens 2004;22:605-10.

32 Anand DV, Lim E, Hopkins D, et al. Risk stratification in uncomplicated type 2 diabetes: prospective evaluation of the combined use of coronary artery calcium imaging and selective myocardial perfusion scintigraphy. Eur Heart J 2006;27:713-21.

33 Sverzellati N, Cademartiri F, Bravi F, et al. Relationship and prognostic value of modified coronary artery calcium score, FEV1, and emphysema in lung cancer screening population: the MILD trial. Radiology 2012;262:460-7.

34 Knez A, Becker A, Leber A, et al. Relation of coronary calcium scores by electron beam tomography to obstructive disease in 2,115 symptomatic patients. Am J Cardiol 2004;93:1150-2

35 Yoo DH, Chun EJ, Choi SI, et al. Significance of noncalcified coronary plaque in asymptomatic subjects with low coronary artery calcium score: assessment with coronary computed tomography angiography. Int J Cardiovasc Imaging 2011;27 (Suppl 1):27-35.

36 McCollough $\mathrm{CH}$, Ulzheimer S, Halliburton SS, et al. Coronary artery calcium : a multi-institutional, multimanufacturer international standard for quantification at cardiac CT. Radiology 2007;243:527-38

37 Budoff MJ, Nasir K, Kinney GL, et al. Coronary artery and thoracic calcium on noncontrast thoracic CT scans: comparison of ungated and gated examinations in patients from the COPD Gene cohort. J Cardiovasc Comput Tomogr 2010:5:1-6.

38 Kirsch J, Buitrago I, Mohammed T-LH, et al. Detection of coronary calcium during standard chest computed tomography correlates with multi-detector computed tomography coronary artery calcium score. Inter I Cardiovasc Imaging 2011;28:1249-56. 